
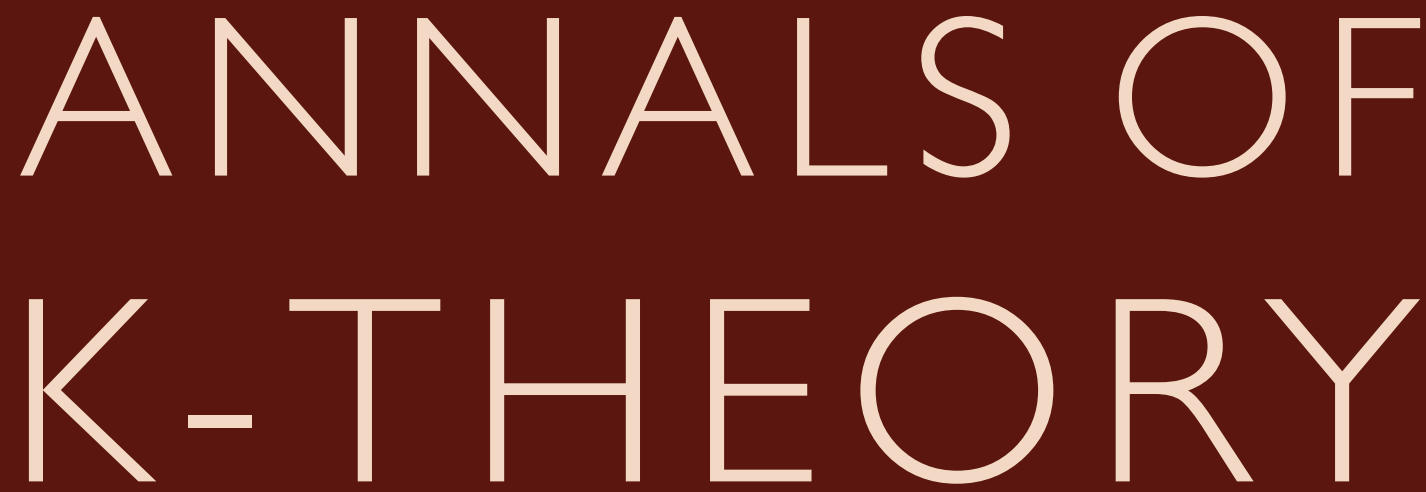

vol. 1 no. 42016

Standard norm varieties for Milnor symbols $\bmod p$

Dinh Huu Nguyen

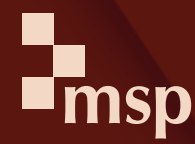

A JOURNAL OF THE K-THEORY FOUNDATION 


\title{
Standard norm varieties for Milnor symbols mod $p$
}

\author{
Dinh Huu Nguyen
}

\begin{abstract}
We prove that the standard norm varieties for Milnor symbols mod $p$ of length $n$ are birationally isomorphic to Pfister quadrics when $p=2$, to Severi-Brauer varieties when $p>2$ and $n=2$, and to varieties defined by reduced norms of cyclic algebras when $p>2$ and $n=3$. In the case $p=2$ and the case $p>2$ and $n=2$, the results imply that the standard norm varieties for two equal Milnor symbols mod $p$ are birationally isomorphic, and we conjecture this in general.
\end{abstract}

\section{Introduction}

The norm residue theorem relates the Milnor K-theory $\bmod p$ of a field $k$ with the étale cohomology of $k$ with coefficients in the twists of $\mu_{p}$. More precisely, it states that for each prime $p \neq \operatorname{char}(k)$ and each weight $n \geq 0$ there exists an isomorphism

$$
K_{n}^{M}(k) / p \cong H_{\mathrm{et}}^{n}\left(k, \mu_{p}^{n}\right)
$$

In 1996, V. Voevodsky [2003] proved the special case of $p=2$, known as the Milnor conjecture. He later [2011] proved the general case of the norm residue theorem, also known as the Bloch-Kato conjecture. His proof used a splitting variety with certain properties for a given Milnor symbol $\left\{a_{1}, \ldots, a_{n}\right\}$ in $K_{n}^{M}(k) / p$. One construction for such splitting varieties was provided by M. Rost in [Haesemeyer and Weibel 2009, Section 3]. Another construction for these varieties was suggested by Voevodsky in [Suslin and Joukhovitski 2006, Section 2]. The entire theorem has been written in book form by C. Haesemeyer and C. Weibel [2016].

In Section 2, we summarize Voevodsky's construction. It uses symmetric powers and produces what are called standard norm varieties.

In Section 3, we show in Theorem 3.7 that the standard norm varieties are birationally isomorphic to Pfister quadrics defined by subforms of Pfister forms when $p=2$. Then we combine this result with the chain P-equivalence theorem

MSC2010: 14E99, 19E99.

Keywords: algebraic geometry, Milnor K-theory, Milnor symbols, norm varieties, standard norm varieties, generic splitting varieties, $p$-generic splitting varieties, norm variety, standard norm variety, generic splitting variety, $p$-generic splitting variety. 
by R. Elman and T. Y. Lam [1972, Main Theorem 3.2] and properties of quadratic forms to prove that the standard norm varieties for two equal symbols are birationally isomorphic in Corollary 3.13.

In Section 4, we use Galois descent to show in Theorem 4.1 that the standard norm varieties are birationally isomorphic to Severi-Brauer varieties when $p>2$ and $n=2$ and get the similar Corollary 4.2.

In Section 5, we use Galois descent to show in Theorem 5.1 that the standard norm varieties are birationally isomorphic to varieties defined by reduced norms of cyclic algebras when $p>2$ and $n=3$. N. Karpenko and A. Merkurjev [2013] use this result and induction to prove $A$-triviality for standard norm varieties.

Given the above two corollaries, we make the following conjecture:

Conjecture 1.1. The standard norm varieties for $\left\{a_{1}, \ldots, a_{n}\right\}$ and $\left\{b_{1}, \ldots, b_{n}\right\}$ are birationally isomorphic if $\left\{a_{1}, \ldots, a_{n}\right\}=\left\{b_{1}, \ldots, b_{n}\right\}$ in $K_{n}^{M}(k) / p$ for all $p$ and $n$.

\section{Symmetric powers}

A general reference for Milnor K-theory is [Milnor 1970]. Throughout this paper, $p$ is a prime and $k$ is a base field of characteristic 0 containing the $p$-th roots of unity. Associated to each nontrivial Milnor symbol $\left\{a_{1}, \ldots, a_{n}\right\}$ in $K_{n}^{M}(k) / p$ are the following notions:

Definition 2.1. A field extension $L / k$ is called a splitting field for $\left\{a_{1}, \ldots, a_{n}\right\}$ if $\left\{a_{1}, \ldots, a_{n}\right\}=0$ in $K_{n}^{M}(L) / p$.

Definition 2.2. A smooth variety $X$ is called a splitting variety for $\left\{a_{1}, \ldots, a_{n}\right\}$ if its function field $k(X)$ is a splitting field for $\left\{a_{1}, \ldots, a_{n}\right\}$. In addition, it is called a generic splitting variety for $\left\{a_{1}, \ldots, a_{n}\right\}$ if any splitting field $L$ for $\left\{a_{1}, \ldots, a_{n}\right\}$ has a point in $X$, i.e., if there exists a morphism $\operatorname{Spec}(L) \rightarrow X$ over $k$.

Such generic splitting varieties are known to exist for all $n$ when $p=2$ and only for $n \leq 3$ when $p>2$. However, if $L^{\prime} / L$ if a finite extension of degree prime to $p$ and $L^{\prime}$ splits $\left\{a_{1}, \ldots, a_{n}\right\}$, then $L$ also splits $\left\{a_{1}, \ldots, a_{n}\right\}$ (using transfer and norm maps). Therefore we can relax our last definition.

Definition 2.3. A smooth variety $X$ is called a p-generic splitting variety for $\left\{a_{1}, \ldots, a_{n}\right\}$ if it is a splitting variety for $\left\{a_{1}, \ldots, a_{n}\right\}$ and, for any splitting field $L$ for $\left\{a_{1}, \ldots, a_{n}\right\}$, there exists an extension $L^{\prime} / L$ of degree prime to $p$ with a point in $X$. In addition, it is called a norm variety for $\left\{a_{1}, \ldots, a_{n}\right\}$ if it is projective and geometrically irreducible of dimension $p^{n-1}-1$.

Example 2.4. When $n=1$, a norm variety for $\left\{a_{1}\right\}$ is $\operatorname{Spec}(L)$, where $L=k\left(\sqrt[p]{a_{1}}\right)$. When $n=2$, a norm variety for $\left\{a_{1}, a_{2}\right\}$ is the Severi-Brauer variety $\mathrm{SB}(A)$ associated to the cyclic algebra $A=\left(a_{1}, a_{2}, \zeta_{p}\right)_{k}$. 
We now describe a standard way to produce these norm varieties for all $n$, which are called standard norm varieties.

Let $X$ be a smooth, quasiprojective, geometrically irreducible variety. The symmetric group $S_{p}$ acts on the product $X^{p}$ and induces the quotient variety $S^{p}(X)$. This quotient variety is geometrically irreducible and normal. Note that $S_{p}$ acts freely on $X^{p} \backslash \triangle$ and $U:=\left(X^{p} \backslash \triangle\right) / S_{p}$ is an open subset in $S^{p}(X)$, where $\triangle$ is the union of all diagonals in $X^{p}$.

For every normal and irreducible scheme $Y$, the set of morphisms $\operatorname{Hom}\left(Y, S^{p}(X)\right)$ can be identified with the set of all effective cycles $Z \subset X \times Y$ such that each component of $Z$ is finite surjective over $Y$, and that the degree of $Z$ over $Y$ is $p$. In particular, the identity map $S^{p}(X) \stackrel{\text { id }}{\longrightarrow} S^{p}(X)$ corresponds to the incidence cycle $Z \subset X \times S^{p}(X)$. In fact, $Z$ is a closed subscheme: it is the image of the closed embedding $X \times S^{p-1}(X) \hookrightarrow X \times S^{p}(X),(x, y) \mapsto(x, x+y)$. Compose this with projection onto the second factor and we get a map

$$
p: X \times S^{p-1}(X) \rightarrow X \times S^{p}(X) \rightarrow S^{p}(X) .
$$

It is finite surjective of degree $p$. Thus we get a diagram

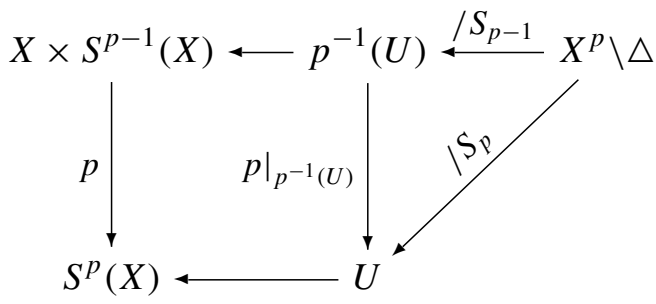

We see that both maps from $X^{p} \backslash \triangle$ are Galois étale coverings, $\left.p\right|_{p^{-1}(U)}$ is a finite étale map of degree $p$, and $U$ is smooth. Furthermore $p_{*} f\left(\mathcal{O}_{X \times S^{p-1}(X)}\right)$ is a coherent $\mathcal{O}_{S^{p}(X)}$-algebra and the sheaf $\mathcal{A}:=p_{*}\left(\left.\mathcal{O}_{X \times S^{p-1}(X)}\right|_{p^{-1}(U)}\right)$ is a locally free $\mathcal{O}_{U}$-algebra of rank $p$. This latter sheaf corresponds to the vector bundle $V:=$ $\operatorname{Spec}\left(S^{\bullet} \mathcal{A}^{v}\right)$ of rank $p$ over $U$. Here $\mathcal{A}^{v}$ denotes the dual of $\mathcal{A}$ and $S^{\bullet} \mathcal{A}^{v}$ denotes its symmetric algebra. There is a well-defined norm map $\mathcal{A} \stackrel{N}{\longrightarrow} \mathcal{O}_{U}$. Locally $N$ is a homogeneous polynomial of degree $p$, that is, $N \in S^{p}\left(\mathcal{A}^{v}\right)$.

A norm variety $X\left(a_{1}, \ldots, a_{n}\right)$ for $\left\{a_{1}, \ldots, a_{n}\right\}$ is then constructed by induction. For $n=2$, we take $X=X\left(a_{1}, a_{2}\right)$ in the preceding construction to be the SeveriBrauer variety $\operatorname{SB}(A)$ associated to the cyclic algebra $A=\left(a_{1}, a_{2}, \zeta_{p}\right)_{k}$. Suppose we have constructed a norm variety $X\left(a_{1}, \ldots, a_{n-1}\right)$ for $\left\{a_{1}, \ldots, a_{n-1}\right\}$. Again let that be $X$ and let $W \subset V$ be the hypersurface defined by the equation $N-a_{n}=0$. By construction, $W$ has dimension $p^{n-1}-1$. By [Suslin and Joukhovitski 2006, Lemma 2.1] it is smooth over $U$ (hence smooth) and geometrically irreducible. By resolution of singularities we can embed $W$ as an open subvariety of a new smooth, projective, geometrically irreducible variety $X^{\prime}$ of the same dimension. Together 
[Suslin and Joukhovitski 2006, Lemma 2.3 and Proposition 2.4] and its subsequent argument show this $X^{\prime}$ is a $p$-generic splitting variety for $\left\{a_{1}, \ldots, a_{n}\right\}$. Hence $X^{\prime}$ is the norm variety that we seek. Note that its construction depends solely on the tuple $\left(a_{1}, \ldots, a_{n}\right)$.

Remark 2.5. The inductive construction could in fact start with $n=1$. We describe explicitly what happens at this stage. Take $X=X\left(a_{1}\right)=\operatorname{Spec}(L)$, where $L=k\left(\sqrt[p]{a_{1}}\right)$. If $\bar{k}$ is the separable closure of $k$ then $\bar{X}=X \times_{\operatorname{Spec}(k)} \operatorname{Spec}(\bar{k})$ has $p$ points; call them $1,2, \ldots, p-1, p$. From there,

$$
\begin{aligned}
& \bar{X}^{p}=\{\text { points on the diagonals }\} \\
& \qquad\left\{\left(n_{1}, n_{2}, \ldots, n_{p}\right) \mid 1 \leq n_{i} \leq p \text { and } n_{i} \neq n_{j} \text { for all } i, j\right\},
\end{aligned}
$$$$
S^{p}(\bar{X})=\bar{X}^{p} / S_{p}=\{\text { classes of points on the diagonals }\} \sqcup\{\overline{(1,2, \ldots, p)}\},
$$$$
\bar{X}^{p} \backslash \triangle=\left\{\left(n_{1}, n_{2}, \ldots, n_{p}\right) \mid 1 \leq n_{i} \leq p \text { and } n_{i} \neq n_{j} \text { for all } i, j\right\},
$$$$
\left(\bar{X}^{p} \backslash \triangle\right) / S_{p}=\{\overline{(1,2, \ldots, p)}\} .
$$

The above square thus looks like this:

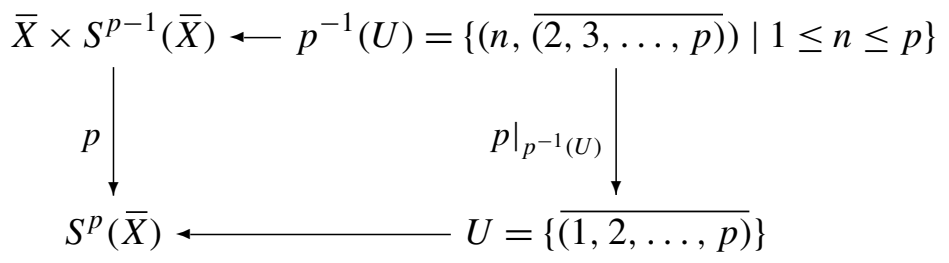

Over $k$ it looks like this:

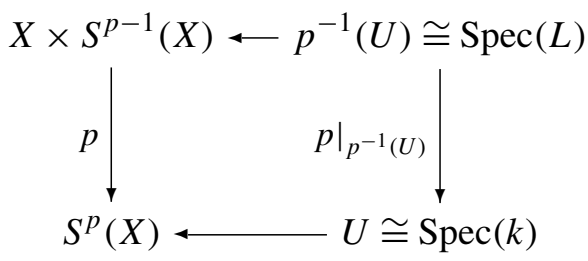

We will use this in Theorems 3.7 and 4.1.

Remark 2.6. Since our problem only concerns birational isomorphism, we can always replace our varieties with birationally isomorphic ones when it suits our purpose but does not change our result. Or we can consider what happens with the generic fiber. For example, in Theorem 3.7 we consider the residue field of the generic fiber of the map $p$ in our construction without mentioning $V, W$ and $X^{\prime}$.

\section{When $p=2$, all $n$}

When $p=2$, we show that the standard norm varieties are birationally isomorphic to Pfister quadrics associated to Pfister forms. This result together with the chain 
P-equivalence theorem and properties of quadratic forms will allow us to compare the standard norm varieties for two equal symbols.

For a quadratic form $\varphi$, let $A_{\varphi}$ denote its symmetric matrix and $D_{k}(\varphi) \subseteq k$ denote the set of its values. Also, for an $n$-tuple $\left(a_{1}, \ldots, a_{n}\right)$, let $\varphi_{n}$ denote the $n$-fold Pfister form $\left\langle\left\langle a_{1}, \ldots, a_{n}\right\rangle\right\rangle=\prod_{i=1}^{n}\left\langle 1,-a_{i}\right\rangle$. Furthermore, we associate to $\varphi_{n}$ the subform $\psi_{n}=\left\langle\left\langle a_{1}, \ldots, a_{n-1}\right\rangle\right\rangle \perp\left\langle-a_{n}\right\rangle$ and denote the quadric defined by $\psi_{n}$ as $Z\left(\psi_{n}\right)$, known as a Pfister quadric. Below are a few more definitions. A general reference for quadratic forms is [Lam 2005].

Definition 3.1. Two quadratic forms $\varphi$ and $\varphi^{\prime}$ are said to be equivalent, written $\varphi \cong \varphi^{\prime}$, if there exists a matrix $C \in \mathrm{GL}(k)$ such that $A_{\varphi^{\prime}}=C A_{\varphi} C^{t}$.

Definition 3.2. Two Pfister forms $\varphi=\left\langle\left\langle a_{1}, \ldots, a_{n}\right\rangle\right\rangle$ and $\varphi^{\prime}=\left\langle\left\langle a_{1}^{\prime}, \ldots, a_{n}^{\prime}\right\rangle\right\rangle$ are said to be simply P-equivalent if there exist two indices $i$ and $j$ such that $\left\langle\left\langle a_{i}, a_{j}\right\rangle\right\rangle \cong$ $\left\langle\left\langle a_{i}^{\prime}, a_{j}^{\prime}\right\rangle\right\rangle$ and $a_{k}=a_{k}^{\prime}$ for $k \neq i, j$. More generally, they are said to be chain P-equivalent, written $\varphi \approx \varphi^{\prime}$, if there exists a sequence $\varphi_{0}, \varphi_{1}, \ldots, \varphi_{m-1}, \varphi_{m}$ of Pfister forms such that $\varphi=\varphi_{0}, \varphi^{\prime}=\varphi_{m}$ and $\varphi_{i}$ is simply P-equivalent to $\varphi_{i+1}$ for $0 \leq i \leq m-1$.

Clearly $\varphi \cong \varphi^{\prime}$ implies $\varphi \cong \varphi^{\prime}$. The converse statement was proven by Elman and Lam [1972] and is called the chain P-equivalence theorem. We recall the statement here, for use in Proposition 3.10.

Theorem 3.3 (chain P-equivalence theorem). Let $\varphi$ and $\varphi^{\prime}$ be $n$-fold Pfister forms. Then $\varphi \cong \varphi^{\prime}$ if and only if $\varphi \cong \varphi^{\prime}$.

Definition 3.4. Two quadratic forms $\varphi$ and $\varphi^{\prime}$ are said to be birationally equivalent if the quadrics they define are birationally isomorphic, i.e., if the function fields $k(Z(\varphi))$ and $k\left(Z\left(\varphi^{\prime}\right)\right)$ are isomorphic.

We begin with a lemma about two equivalent Pfister forms and the matrix that connects them.

Lemma 3.5. If $\varphi_{n-1}$ and $\varphi_{n}=\langle 1,-b\rangle \varphi_{n-1}$ are Pfister forms with matrices $A_{\varphi_{n-1}}$ and $A_{\varphi_{n}}$, and $c=\varphi_{n}\left(x_{1}, \ldots, x_{2^{n}}\right)$, then $\varphi_{n} \cong\langle c\rangle \varphi_{n}$ via a matrix

$$
C_{n} \in \mathrm{GL}_{2^{n}}\left(k\left(x_{1}, \ldots, x_{2^{n}}\right)\right)
$$

— that is, $C_{n} A_{\varphi_{n}} C_{n}^{t}=c A_{\varphi_{n}}$ - which satisfies two properties:

(1) $C_{n}^{-1}=C_{n} / c$, hence $\left(C_{n}^{t}\right)^{-1}=C_{n}^{t} / c$ as well.

(2) The first row and first column of $C_{n}$ are $\left(x_{1} \cdots x_{2^{n}}\right)$ and $A_{\varphi_{n}}\left(x_{1} \cdots x_{2^{n}}\right)^{t}$.

Proof. We induce on $n$. For $n=1$ and $c=x_{1}^{2}-a x_{2}^{2}$, we have $\varphi_{1} \cong c \varphi_{1}$ via

$$
C_{1}=\left(\begin{array}{rr}
x_{1} & x_{2} \\
-a x_{2} & -x_{1}
\end{array}\right) \text {, }
$$


which satisfies (1) and (2).

Next, write

$$
A_{\varphi_{n}}=\left(\begin{array}{cc}
A_{\varphi_{n-1}} & 0 \\
0 & -b A_{\varphi_{n-1}}
\end{array}\right)
$$

then $c=\varphi_{n}\left(x_{1}, \ldots, x_{2^{n}}\right)=x A_{\varphi_{n}} x^{t}=s-b t \in D_{k}\left(\varphi_{n}\right)$, where $s=\varphi_{n-1}\left(x_{1}, \ldots, x_{2^{n-1}}\right)$ and $t=\varphi_{n-1}\left(x_{2^{n-1}+1}, \ldots, x_{2^{n}}\right)$ are in $D_{k}\left(\varphi_{n-1}\right)$. By induction, $\varphi_{n-1} \cong\langle s\rangle \varphi_{n-1}$ via a matrix $C \in \mathrm{GL}_{2^{n-1}}\left(k\left(x_{1}, \ldots, x_{2^{n-1}}\right)\right)$, that is, $C A \varphi_{n-1} C^{t}=s A_{\varphi_{n-1}}$, which satisfies:

(1) $C^{-1}=C / s$, hence $\left(C^{t}\right)^{-1}=C^{t} / s$.

(2) The first row and first column of $C$ are $\left(x_{1} \cdots x_{2^{n-1}}\right)$ and $A_{\varphi_{n-1}}\left(x_{1} \cdots x_{2^{n-1}}\right)^{t}$.

Similarly, $\varphi_{n-1} \cong\langle t\rangle \varphi_{n-1}$ via $C^{\prime} \in \mathrm{GL}_{2^{n-1}}\left(F\left(x_{2^{n-1}+1}, \ldots, x_{2^{n}}\right)\right)$ with the same properties. From this, we have:

(i) $\varphi_{n} \cong\langle s\rangle \varphi_{n-1} \perp\langle-b\rangle\langle t\rangle \varphi_{n-1}=\langle s,-b t\rangle \varphi_{n-1}$ with

$$
\left(\begin{array}{cc}
C & 0 \\
0 & C^{\prime}
\end{array}\right)\left(\begin{array}{cc}
A_{\varphi_{n-1}} & 0 \\
0 & -b A_{\varphi_{n-1}}
\end{array}\right)\left(\begin{array}{cc}
C^{t} & 0 \\
0 & C^{\prime t}
\end{array}\right)=\left(\begin{array}{cc}
s A_{\varphi_{n-1}} & 0 \\
0 & -b t A_{\varphi_{n-1}}
\end{array}\right) .
$$

(ii) $\langle s,-b t\rangle \varphi_{n-1} \cong\langle c,-c b s t\rangle \varphi_{n-1}$ with

$$
\left(\begin{array}{cc}
I & I \\
b t I & s I
\end{array}\right)\left(\begin{array}{cc}
s A_{\varphi_{n-1}} & 0 \\
0 & -b t A_{\varphi_{n-1}}
\end{array}\right)\left(\begin{array}{cc}
I & b t I \\
I & s I
\end{array}\right)=\left(\begin{array}{cc}
c A_{\varphi_{n-1}} & 0 \\
0 & -\operatorname{cbst} A_{\varphi_{n-1}}
\end{array}\right) .
$$

(iii) Let $D=\left(C C^{\prime}\right)^{-1}=C^{\prime-1} C^{-1}=C^{\prime} C / t s$; then

$$
\langle c,-c b s t\rangle \varphi_{n-1} \cong\langle c,-c b\rangle \varphi_{n-1}=\langle c\rangle \varphi_{n}
$$

with

$$
\begin{aligned}
\left(\begin{array}{ll}
I & 0 \\
0 & D
\end{array}\right)\left(\begin{array}{cc}
c A_{\varphi_{n-1}} & 0 \\
0 & -c b s t A_{\varphi_{n-1}}
\end{array}\right)\left(\begin{array}{cc}
I & 0 \\
0 & D^{t}
\end{array}\right) \\
=\left(\begin{array}{cc}
c A_{\varphi_{n-1}} & 0 \\
0 & -c b s t D A_{\varphi_{n-1}}
\end{array}\right)\left(\begin{array}{cc}
I & 0 \\
0 & D^{t}
\end{array}\right) \\
=\left(\begin{array}{cc}
c A_{\varphi_{n-1}} & 0 \\
0 & -c b s t D A_{\varphi_{n-1}} D^{t}
\end{array}\right) \\
=\left(\begin{array}{cc}
c A_{\varphi_{n-1}} & 0 \\
0 & -c b s t A_{\varphi_{n-1}} / s t
\end{array}\right) \\
=\left(\begin{array}{cc}
c A_{\varphi_{n-1}} & 0 \\
0 & -c b A_{\varphi_{n-1}}
\end{array}\right) .
\end{aligned}
$$

(iv) Putting (i), (ii) and (iii) together, we get $\varphi_{n} \cong\langle s\rangle \varphi_{n-1} \perp\langle-b\rangle\langle t\rangle \varphi_{n-1}=$ $\langle s,-b t\rangle \varphi_{n-1} \cong\langle c,-c b s t\rangle \varphi_{n-1} \cong\langle c,-c b\rangle \varphi_{n-1}=\langle c\rangle \varphi_{n}$ via $C_{n}^{\prime}$, where 


$$
\begin{aligned}
& C_{n}^{\prime}=\left(\begin{array}{ll}
I & 0 \\
0 & D
\end{array}\right)\left(\begin{array}{cc}
I & I \\
b t I & s I
\end{array}\right)\left(\begin{array}{cc}
C & 0 \\
0 & C^{\prime}
\end{array}\right) \\
& =\left(\begin{array}{cc}
I & I \\
b t D & s D
\end{array}\right)\left(\begin{array}{cc}
C & 0 \\
0 & C^{\prime}
\end{array}\right) \\
& =\left(\begin{array}{cc}
C & C^{\prime} \\
b t C^{\prime-1} C^{-1} C & s C^{\prime-1} C^{-1} C^{\prime}
\end{array}\right) \\
& =\left(\begin{array}{cc}
C & C^{\prime} \\
b C^{\prime} & C^{\prime} C C^{\prime} / t
\end{array}\right) \text {. }
\end{aligned}
$$

Finally, let

$$
C_{n}=\left(\begin{array}{cc}
I & 0 \\
0 & -I
\end{array}\right) C_{n}^{\prime}=\left(\begin{array}{cc}
C & C^{\prime} \\
-b C^{\prime} & -C^{\prime} C C^{\prime} / t
\end{array}\right)
$$

then its inverse $C_{n}^{-1}$ equals $C_{n} / c$, its first row and first column are $\left(x_{1} \cdots x_{2^{n}}\right)$ and $A_{\varphi_{n}}\left(x_{1} \cdots x_{2^{n}}\right)^{t}$, and

$$
C_{n} A_{\varphi_{n}} C_{n}^{t}=\left(\begin{array}{rr}
I & 0 \\
0 & -I
\end{array}\right) C_{n}^{\prime} A_{\varphi_{n}} C_{n}^{\prime t}\left(\begin{array}{rr}
I & 0 \\
0 & -I
\end{array}\right)=c A_{\varphi_{n}} .
$$

The last equality can be verified directly:

$C_{n} A_{\varphi_{n}} C_{n}^{t}$

$=\left(\begin{array}{cc}C & C^{\prime} \\ -b C^{\prime} & -C^{\prime} C C^{\prime} / t\end{array}\right)\left(\begin{array}{cc}A_{\varphi_{n-1}} & 0 \\ 0 & -b A_{\varphi_{n-1}}\end{array}\right)\left(\begin{array}{cc}C^{t} & -b C^{\prime t} \\ C^{\prime t} & -C^{\prime t} C^{t} C^{\prime t} / t\end{array}\right)$

$=\left(\begin{array}{cc}C A_{\varphi_{n-1}} & -b C^{\prime} A_{\varphi_{n-1}} \\ -b C^{\prime} A_{\varphi_{n-1}} & (b / t) C^{\prime} C C^{\prime} A_{\varphi_{n-1}}\end{array}\right)\left(\begin{array}{cc}C^{t} & -b C^{\prime t} \\ C^{\prime t} & -C^{\prime t} C^{t} C^{\prime t} / t\end{array}\right)$

$=\left(\begin{array}{cc}C A_{\varphi_{n-1}} C^{t}-b C^{\prime} A_{\varphi_{n-1}} C^{\prime t} & -b C A_{\varphi_{n-1}} C^{\prime t}+(b / t) C^{\prime} A_{\varphi_{n-1}} C^{\prime t} C^{t} C^{\prime t} \\ -b C^{\prime} A_{\varphi_{n-1}} C^{t}+(b / t) C^{\prime} C C^{\prime} A_{\varphi_{n-1}} C^{\prime t} & b^{2} C^{\prime} A_{\varphi_{n-1}} C^{\prime t}-\left(b / t^{2}\right) C^{\prime} C C^{\prime} A_{\varphi_{n-1}} C^{\prime t} C^{t} C^{\prime t}\end{array}\right)$

$=\left(\begin{array}{cc}s A_{\varphi_{n-1}}-b t A_{\varphi_{n-1}} & -b C A_{\varphi_{n-1}} C^{\prime t}+b A_{\varphi_{n-1}} C^{t} C^{\prime t} \\ -b C^{\prime} A_{\varphi_{n-1}} C^{t}+b C^{\prime} C A_{\varphi_{n-1}} & b^{2} t A_{\varphi_{n-1}}-b s A_{\varphi_{n-1}}\end{array}\right)$

$=\left(\begin{array}{cc}c A_{\varphi_{n-1}} & -b C A_{\varphi_{n-1}} C^{\prime t}+(b / s) C A_{\varphi_{n-1}} C^{t} C^{t} C^{\prime t} \\ -b C^{\prime} A_{\varphi_{n-1}} C^{t}+(b / s) C^{\prime} C C A_{\varphi_{n-1}} C^{t} & -b c A_{\varphi_{n-1}}\end{array}\right)$

$=\left(\begin{array}{cc}c A_{\varphi_{n-1}} & -b C A_{\varphi_{n-1}} C^{\prime t}+b C A_{\varphi_{n-1}} C^{\prime t} \\ -b C^{\prime} A_{\varphi_{n-1}} C^{t}+b C^{\prime} A_{\varphi_{n-1}} C^{t} & -b c A_{\varphi_{n-1}}\end{array}\right)$

$=\left(\begin{array}{cc}c A_{\varphi_{n-1}} & 0 \\ 0 & -b c A_{\varphi_{n-1}}\end{array}\right)$

$=c A_{\varphi_{n}}$.

This concludes the proof. 
The next lemma is needed to show that the residue field in Theorem 3.7 stays the same.

Lemma 3.6. The $n \times n$ matrix

$$
M=\left(\begin{array}{cccc}
a_{1} b_{1} & a_{1} b_{2} & \cdots & a_{1} b_{n} \\
a_{2} b_{1} & a_{2} b_{2} & \cdots & a_{2} b_{n} \\
\vdots & \vdots & \ddots & \vdots \\
a_{n} b_{1} & a_{n} b_{2} & \cdots & a_{n} b_{n}
\end{array}\right)
$$

has characteristic polynomial $\operatorname{char}_{M}(t)=t^{n-1}\left(t-a_{1} b_{1}-a_{2} b_{2}-\cdots-a_{n} b_{n}\right)$.

Proof. We consider what $M$ does to the standard basis:

$$
\begin{aligned}
k^{n} & \stackrel{M}{\longmapsto} k^{n}, \\
(1,0, \ldots, 0) & \longmapsto b_{1}\left(a_{1}, \ldots, a_{n}\right), \\
(0,1, \ldots, 0) & \longmapsto b_{2}\left(a_{1}, \ldots, a_{n}\right), \\
& \vdots \\
(0,0, \ldots, 1) & \longmapsto b_{n}\left(a_{1}, \ldots, a_{n}\right) .
\end{aligned}
$$

Thus $M$ sends $\left(a_{1}, \ldots, a_{n}\right)$ to $\alpha\left(a_{1}, \ldots, a_{n}\right)$, where $\alpha=a_{1} b_{1}+a_{2} b_{2}+\cdots+a_{n} b_{n}$. Letting $v_{1}=\left(a_{1}, \ldots, a_{n}\right)$, we choose a new basis $\left\{v_{1}, \ldots, v_{n}\right\}$ for $k^{n}$ such that $\operatorname{ker}(M)=\left\langle v_{2}, \ldots, v_{n}\right\rangle$ and again look at what $\mathrm{M}$ does as a linear map:

$$
\begin{aligned}
k^{n} & \stackrel{M}{\longmapsto} k^{n}, \\
v_{1} \longmapsto & \longmapsto, 0, \ldots, 0), \\
v_{2} \longmapsto(0, \ldots, 0), & \\
& \vdots \\
v_{n} \longmapsto & \longmapsto, \ldots, 0) .
\end{aligned}
$$

Therefore $\mathrm{M}$ has canonical form

$$
\left(\begin{array}{cccc}
\alpha & 0 & \cdots & 0 \\
0 & 0 & \cdots & 0 \\
\vdots & \vdots & \ddots & \vdots \\
0 & 0 & \cdots & 0
\end{array}\right)
$$

and $\operatorname{det}(t I-M)=\operatorname{char}_{M}(t)=t^{n}-\alpha t^{n-1}=t^{n-1}\left(t-a_{1} b_{1}-a_{2} b_{2}-\cdots-a_{n} b_{n}\right)$.

We are now ready to turn the standard norm varieties into Pfister quadrics defined by subforms of Pfister forms.

Theorem 3.7. The standard norm variety $X\left(a_{1}, \ldots, a_{n}\right)$ for $\left\{a_{1}, \ldots, a_{n}\right\}$ is birationally isomorphic to the Pfister quadric $Z\left(\psi_{n}\right) \subset \mathbb{P}_{k}^{2^{n-1}}$ defined by the subform $\psi_{n}=\left\langle\left\langle a_{1}, \ldots, a_{n-1}\right\rangle\right\rangle \perp\left\langle-a_{n}\right\rangle$ of the Pfister form $\varphi_{n}=\left\langle\left\langle a_{1}, \ldots, a_{n}\right\rangle\right\rangle$. 
Proof. We induce on $n$. First we verify the case $n=2$. As described in Remark 2.5, we begin our symmetric power construction with $X\left(a_{1}\right)=\operatorname{Spec}(L)$, where $L=$ $k\left(\sqrt{a_{1}}\right)$ and get

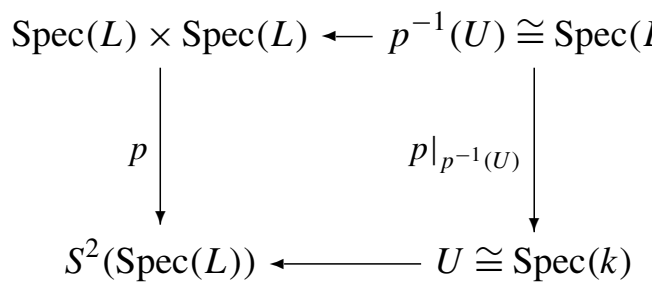

Hence $X\left(a_{1}, a_{2}\right)=Z\left(N_{L / k}-a_{2}\right)=Z\left(x_{1}^{2}-a_{1} x_{2}^{2}-a_{2}\right)$, the hypersurface defined by the equation $N_{L / k}-a_{2}=x_{1}^{2}-a_{1} x_{2}^{2}-a_{2}=0$. Projectivization then gives $X\left(a_{1}, a_{2}\right)=Z\left(x_{1}^{2}-a_{1} x_{2}^{2}-a_{2} x_{3}^{2}\right)=Z\left(\psi_{2}\right) \subset \mathbb{P}_{k}^{2}$, as required.

By induction, $X\left(a_{1}, \ldots, a_{n+1}\right) \approx Z\left(\psi_{n+1}\right)$. Write $\psi=\psi_{n+1}=\varphi_{n} \perp\left\langle-a_{n+1}\right\rangle=$ $\langle 1\rangle \perp \varphi^{\prime} \perp\left\langle-a_{n+1}\right\rangle \cong\left\langle 1,-a_{n+1}\right\rangle \perp \varphi^{\prime}$, where $\varphi^{\prime}$ is the pure subform of $\varphi$. By construction, we get

$$
\left(X_{n+1} \times X_{n+1}\right) \backslash \triangle \longrightarrow\left(\left(X_{n+1} \times X_{n+1}\right) \backslash \triangle\right) / s_{2} \longrightarrow \operatorname{Gr}\left(2, \mathbb{A}_{k}^{2^{n}+1}\right) .
$$

Let $U=\langle u, v\rangle=\left\langle\left(1,0, x_{2}, \ldots, x_{2^{n}}\right),\left(0,1, y_{2}, \ldots, y_{2^{n}}\right)\right\rangle$ be the generic plane in $\mathbb{A}_{k}^{2^{n}+1}$ and moreover let $\{u, v\}$ be a basis for $U$. Over this basis, the restriction $\left.\psi_{k\left(x_{i}, y_{i}\right)}\right|_{U}$ has matrix form

$$
\left(\begin{array}{cc}
\psi(u) & b(u, v) \\
b(u, v) & \psi(v)
\end{array}\right)
$$

where

$$
U \times U \stackrel{b}{\longrightarrow} k, \quad\left(u^{\prime}, v^{\prime}\right) \mapsto \frac{1}{2}\left(\psi\left(u^{\prime}+v^{\prime}\right)-\psi\left(u^{\prime}\right)-\psi\left(v^{\prime}\right)\right),
$$

is the symmetric bilinear form associated to $\left.\psi_{k\left(x_{i}, y_{i}\right)}\right|_{U}$.

The generic fiber is then the point $(r, s) \in U$ such that

$$
\psi(r, s)=\psi(u, u) r^{2}+2 b(u, v) r s+\psi(v, v) s^{2}=0,
$$

with residue field

$$
q f\left(k\left(x_{i}, y_{i}\right)\left[\frac{r}{s}\right] /\left(\psi(u, u)\left(\frac{r}{s}\right)^{2}+2 b(u, v) \frac{r}{s}+\psi(v, v)\right)\right)=k\left(x_{i}, y_{j}\right)(\sqrt{-\theta}),
$$

where

$$
\begin{aligned}
\theta & =\psi(u) \psi(v)-b(u, v)^{2} \\
& =\left(1+\varphi^{\prime}\left(x_{2}, \ldots, x_{2^{n}}\right)\right)\left(-a_{n+1}+\varphi^{\prime}\left(y_{2}, \ldots, y_{2^{n}}\right)\right)-b(u, v)^{2} \\
& =\left(\varphi\left(1, x_{2}, \ldots, x_{2^{n}}\right)\right)\left(-a_{n+1}+\varphi^{\prime}\left(y_{2}, \ldots, y_{2^{n}}\right)\right)-b(u, v)^{2} \\
& =\left(-a_{n+1}\right) \varphi\left(1, x_{2}, \ldots, x_{2^{n}}\right)+\varphi\left(1, x_{2}, \ldots, x_{2^{n}}\right) \varphi\left(0, y_{2}, \ldots, y_{2^{n}}\right)-b(u, v)^{2} .
\end{aligned}
$$


If we write $\varphi=\left\langle 1, c_{2}, \ldots, c_{2^{n}}\right\rangle$ then, by Lemma 3.5, there exists a matrix

$$
C_{n}=\left(\begin{array}{cccc}
1 & x_{2} & \cdots & x_{2^{n}} \\
c_{2} x_{2} & \ddots & & \\
\vdots & & \ddots & \\
c_{2^{n}} x_{2^{n}} & & & \ddots
\end{array}\right)
$$

such that $\varphi\left(1, x_{2}, \ldots, x_{2^{n}}\right) \varphi\left(0, y_{2}, \ldots, y_{2^{n}}\right)=\varphi\left(\left(0, y_{2}, \ldots, y_{2^{n}}\right) C_{n}\right)$. So

$$
\begin{aligned}
\theta & =\left(-a_{n+1}\right) \varphi\left(1, x_{2}, \ldots, x_{2^{n}}\right)+\varphi\left(\left(0, y_{2}, \ldots, y_{2^{n}}\right) C_{n}\right)-b(u, v)^{2} \\
= & \left(-a_{n+1}\right) \varphi\left(1, x_{2}, \ldots, x_{2^{n}}\right)+\varphi\left(\left(0, y_{2}, \ldots, y_{2^{n}}\right) A_{\varphi}\left(1, x_{2}, \ldots, x_{2^{n}}\right)^{t}, z_{2}, \ldots, z_{2^{n}}\right) \\
& -\left(\left(y_{2}, \ldots, y_{2^{n}}\right) A_{\varphi^{\prime}}\left(x_{2}, \ldots, x_{2^{n}}\right)^{t}\right)^{2} \\
= & \left(-a_{n+1}\right) \varphi\left(1, x_{2}, \ldots, x_{2^{n}}\right)+\varphi\left(\left(y_{2}, \ldots, y_{2^{n}}\right) A_{\varphi^{\prime}}\left(x_{2}, \ldots, x_{2^{n}}\right)^{t}, z_{2}, \ldots, z_{2^{n}}\right) \\
\quad-\left(\left(y_{2}, \ldots, y_{2^{n}}\right) A_{\varphi^{\prime}}\left(x_{2}, \ldots, x_{2^{n}}\right)^{t}\right)^{2} & \\
= & \left(-a_{n+1}\right) \varphi\left(1, x_{2}, \ldots, x_{2^{n}}\right)+\varphi^{\prime}\left(z_{2}, \ldots, z_{2^{n}}\right) .
\end{aligned}
$$

Above, we let $\left(z_{1}, z_{2}, \ldots, z_{2^{n}}\right)=\left(0, y_{2}, \ldots, y_{2^{n}}\right) C_{n}$, so that $\left(z_{2}, \ldots, z_{2^{n}}\right)=$ $\left(y_{2}, \ldots, y_{2^{n}}\right) M$, where $M$ is $C_{n}$ without its first row and first column. Since $C_{n}^{2}=\varphi\left(1, x_{2}, \ldots, x_{2^{n}}\right) I$, it follows that $M^{2}=\varphi\left(1, x_{2}, \ldots, x_{n}\right) I-\left(c_{i} x_{i} x_{j}\right)$ for $2 \leq i, j \leq 2^{n}$. By Lemma 3.6,

$$
\operatorname{det}\left(M^{2}\right)=\varphi\left(1, x_{2}, \ldots, x_{2^{n}}\right)^{2^{n}-2} .
$$

Thus $\operatorname{det}(M)=\varphi\left(1, x_{2}, \ldots, x_{2^{n}}\right)^{2^{n-1}-1}$ and $M \in \operatorname{GL}_{2^{n}-1}\left(F\left(x_{2}, \ldots, x_{2^{n}}\right)\right)$. So the residue field stays the same:

$$
F\left(x_{i}, y_{j}\right)(\sqrt{-\theta})=F\left(x_{i}, z_{j}\right)(\sqrt{-\theta})
$$

It has quadratic norm

$$
\begin{aligned}
N(m+n \sqrt{-\theta}) & =m^{2}-a_{n+1} \varphi\left(1, x_{2}, \ldots, x_{2^{n}}\right) n^{2}+\varphi^{\prime}\left(z_{2}, \ldots, z_{2^{n}}\right) n^{2} \\
& =\varphi\left(m, n z_{2}, \ldots, n z_{2^{n}}\right)-a_{n+1} \varphi\left(n, n x_{2}, \ldots, n x_{2^{n}}\right) \\
& =\left\langle 1,-a_{n+1}\right\rangle \varphi\left(m, n z_{2}, \ldots, n z_{2^{n}}, n, n x_{2}, \ldots, n x_{2^{n}}\right) \\
& =\varphi_{n+1}\left(t_{1}, \ldots, t_{2^{n+1}}\right) .
\end{aligned}
$$

Therefore our projectivized $X\left(a_{1}, \ldots, a_{n+2}\right)=Z\left(N-a_{n+2} t_{2^{n+1}+1}^{2}\right)$ is birationally isomorphic to $Z\left(\varphi_{n+1} \perp\left\langle-a_{n+2}\right\rangle\right)=Z\left(\psi_{n+2}\right) \subset \mathbb{P}_{k}^{2^{n+1}}$, as wanted.

Next, we show that interchanging $a_{i}$ and $a_{j}$ or multiplying $a_{i}$ by any nonzero norm $N_{k\left(\sqrt{a_{j}}\right) / k}(u)$ in the symbol $\left\{a_{1}, \ldots, a_{n}\right\}$ does not change its standard norm variety. For this, we need two more lemmas about Pfister neighbors; the first one we will use toward our Corollary 3.13 and the second one we will use toward our Example 3.14. 
Lemma 3.8. If $\varphi=\left\langle\left\langle a_{1}, \ldots, a_{n}\right\rangle\right\rangle$ is an anisotropic Pfister form then the two forms $\varphi \perp\langle-b \varphi\rangle \perp\langle-c\rangle$ and $\varphi \perp\langle-c \varphi\rangle \perp\langle-b\rangle$ are birationally equivalent.

Proof. We connect the quadrics defined by these two forms by a sequence of birationally isomorphic ones. Let $(x, y, z)$ be the generic zero for the form $\varphi \perp$ $\langle-b \varphi\rangle \perp\langle-c\rangle$; then

$$
\varphi(x)-b \varphi(y)-c z^{2}=0 .
$$

Since $\varphi$ is Pfister and $\varphi(y) \in D_{k(y)}(\varphi)$, it follows $\varphi \cong \varphi(y) \varphi$ over $k(y)$. That means there exists a matrix $C \in \mathrm{GL}(k(y))$ such that $\varphi(x)=\varphi(y) \varphi(C x)$. Let $x^{\prime}=C x$; then $k(x, y, z)=k\left(x^{\prime}, y, z\right)$ and

$$
\varphi(y) \varphi\left(x^{\prime}\right)-b \varphi(y)-c z^{2}=0,
$$

hence

$$
\varphi\left(x^{\prime}\right)-b-c \frac{z^{2}}{\varphi(y)}=0 .
$$

Now let $y^{\prime}=y / \varphi(y)$; then $k(x, y, z)=k\left(x^{\prime}, y^{\prime}, z\right)$ and

$$
\varphi\left(x^{\prime}\right)-b-c z^{2} \varphi\left(y^{\prime}\right)=0,
$$

hence

$$
\frac{\varphi\left(x^{\prime}\right)}{z^{2}}-\frac{b}{z^{2}}-c \varphi\left(y^{\prime}\right)=0 .
$$

Finally, let $x^{\prime \prime}=x^{\prime} / z$ and $z^{\prime}=1 / z$; then $\left(x^{\prime \prime}, y^{\prime}, z^{\prime}\right)$ is a generic zero for $\varphi \perp\langle-c \varphi\rangle \perp\langle-b\rangle, k(x, y, z)=k\left(x^{\prime \prime}, y^{\prime}, z^{\prime}\right)$ and

$$
\varphi\left(x^{\prime \prime}\right)-c \varphi\left(y^{\prime}\right)-b z^{\prime 2}=0 .
$$

Therefore, the two forms $\varphi \perp\langle-b \varphi\rangle \perp\langle-c\rangle$ and $\varphi \perp\langle-c \varphi\rangle \perp\langle-b\rangle$ are birationally equivalent.

Lemma 3.9. If $\varphi=\left\langle\left\langle a_{1}, \ldots, a_{n}\right\rangle\right\rangle$ is an anisotropic Pfister form then the two forms $\varphi \perp\langle-b\rangle$ and $\varphi \perp\left\langle-b \varphi\left(x_{0}\right)\right\rangle$ with $\varphi\left(x_{0}\right) \neq 0$ are birationally equivalent. In particular, $\varphi \perp\langle-b\rangle \approx \varphi \perp\left\langle-b N_{k\left(\sqrt{a_{i}}\right) / k}(u)\right\rangle$ for any nonzero norm $N_{k\left(\sqrt{a_{i}}\right) / k}(u)$.

Proof. We use the same approach as in Lemma 3.8. Let $(x, y)$ be a generic zero for the form $\varphi \perp\left\langle-b \varphi\left(x_{0}\right)\right\rangle$; then

$$
\varphi(x)-b \varphi\left(x_{0}\right) y^{2}=0,
$$

hence

$$
\varphi\left(x_{0}\right) \varphi(x)-b \varphi\left(x_{0}\right)^{2} y^{2}=0 .
$$

Again $\varphi \cong \varphi\left(x_{0}\right) \varphi$ over $k$, i.e., there exists a matrix $C \in \mathrm{GL}(k)$ such that $\varphi(C x)=\varphi\left(x_{0}\right) \varphi(x)$. Let $x^{\prime}=C x$ and $y^{\prime}=\varphi\left(x_{0}\right) y$; then $\left(x^{\prime}, y^{\prime}\right)$ is a generic 
zero for $\varphi \perp\langle-b\rangle, k(x, y)=k\left(x^{\prime}, y^{\prime}\right)$ and

$$
\varphi\left(x^{\prime}\right)-b y^{\prime 2}=0
$$

Therefore, the two forms $\varphi \perp\langle-b\rangle$ and $\varphi \perp\left\langle-b \varphi\left(x_{0}\right)\right\rangle$ with $\varphi\left(x_{0}\right) \neq 0$ are birationally equivalent. The last statement follows when we choose $x_{0}$ such that $\varphi\left(x_{0}\right)=N_{k\left(\sqrt{a_{i}}\right) / k}(u)$.

Proposition 3.10. If two Pfister forms $\varphi$ and $\varphi^{\prime}$ are equivalent then their associated subforms $\psi$ and $\psi^{\prime}$ are birationally equivalent.

Proof. By the chain P-equivalence theorem, $\varphi \simeq \varphi^{\prime}$. So there exists a sequence of Pfister forms $\varphi_{0}, \varphi_{1}, \ldots, \varphi_{t}, \ldots, \varphi_{m-1}, \varphi_{m}$ such that $\varphi=\varphi_{0}, \varphi^{\prime}=\varphi_{m}$ and $\varphi_{t}$ is simply P-equivalent to $\varphi_{t+1}$ for $0 \leq t \leq m-1$. Write $\varphi_{t}=\left\langle\left\langle a_{1}, \ldots, a_{i}, \ldots, a_{j}, \ldots, a_{n}\right\rangle\right\rangle$ and $\varphi_{t+1}=\left\langle\left\langle a_{1}, \ldots, a_{i}^{\prime}, \ldots, a_{j}^{\prime}, \ldots, a_{n}\right\rangle\right\rangle$, where $\left\langle\left\langle a_{i}, a_{j}\right\rangle\right\rangle \cong\left\langle\left\langle a_{i}^{\prime}, a_{j}^{\prime}\right\rangle\right\rangle$. If $i=j$ then there is nothing to do. Otherwise, we consider each case separately:

(1) If $j \neq n$ then

$$
\begin{aligned}
\psi_{t} & =\left\langle\left\langle a_{1}, \ldots, a_{i}, \ldots, a_{j}, \ldots, a_{n-1}\right\rangle\right\rangle \perp\left\langle-a_{n}\right\rangle \\
& \cong\left\langle\left\langle a_{1}, \ldots, a_{i}^{\prime}, \ldots, a_{j}^{\prime}, \ldots, a_{n-1}\right\rangle\right\rangle \perp\left\langle-a_{n}\right\rangle \\
& =\psi_{t+1} .
\end{aligned}
$$

(2) If $j=n$ and $i \neq n-1$ then, by Lemma 3.8,

$$
\begin{aligned}
\psi_{t} & =\left\langle\left\langle a_{1}, \ldots, a_{i}, \ldots, a_{n-1}\right\rangle\right\rangle \perp\left\langle-a_{j}\right\rangle \\
& \approx\left\langle\left\langle a_{1}, \ldots, a_{i}, \ldots, a_{j}\right\rangle\right\rangle \perp\left\langle-a_{n-1}\right\rangle \\
& \cong\left\langle\left\langle a_{1}, \ldots, a_{i}^{\prime}, \ldots, a_{j}^{\prime}\right\rangle\right\rangle \perp\left\langle-a_{n-1}\right\rangle \\
& \approx\left\langle\left\langle a_{1}, \ldots, a_{i}^{\prime}, \ldots, a_{n-1}\right\rangle\right\rangle \perp\left\langle-a_{j}^{\prime}\right\rangle \\
& =\psi_{t+1} .
\end{aligned}
$$

(3) If $j=n$ and $i=n-1$ then, again by Lemma 3.8,

$$
\begin{aligned}
\psi_{t} & =\left\langle\left\langle a_{1}, \ldots, a_{n-2}, a_{i}\right\rangle\right\rangle \perp\left\langle-a_{j}\right\rangle \\
& \cong\left\langle\left\langle a_{1}, \ldots, a_{i}, a_{n-2}\right\rangle\right\rangle \perp\left\langle-a_{j}\right\rangle \\
& \approx\left\langle\left\langle a_{1}, \ldots, a_{i}, a_{j}\right\rangle\right\rangle \perp\left\langle-a_{n-2}\right\rangle \\
& \cong\left\langle\left\langle a_{1}, \ldots, a_{i}^{\prime}, a_{j}^{\prime}\right\rangle\right\rangle \perp\left\langle-a_{n-2}\right\rangle \\
& \approx\left\langle\left\langle a_{1}, \ldots, a_{i}^{\prime}, a_{n-2}\right\rangle\right\rangle \perp\left\langle-a_{j}^{\prime}\right\rangle \\
& \cong\left\langle\left\langle a_{1}, \ldots, a_{n-2}, a_{i}^{\prime}\right\rangle\right\rangle \perp\left\langle-a_{j}^{\prime}\right\rangle \\
& =\psi_{t+1} .
\end{aligned}
$$

Hence $\psi_{t} \approx \psi_{t+1}$ for all $t$, and $\psi \approx \psi^{\prime}$. 
Remark 3.11. Let $\varphi$ be a Pfister form of dimension greater than or equal to 2, $c \in k^{\times}$, and $\varphi_{1}$ a nonzero subform of $\varphi$. In [Ahmad and Ohm 1995], H. Ahmad called $\left(\varphi, c, \varphi_{1}\right)$ a Pfister triple, $\varphi \perp\langle c\rangle$ the base form, $\varphi \perp c \varphi_{1}$ the form defined by the triple, $\varphi \perp c \varphi$ the associated Pfister form, and any form similar to such $\varphi \perp c \varphi_{1}$ a special Pfister neighbor. In this setting the forms in Lemma 3.8 and the forms in Lemma 3.9 are pairwise special Pfister neighbors of the same dimensions and have the same associated Pfister forms $\varphi \otimes\langle\langle b, c\rangle\rangle$ and $\varphi \otimes\langle\langle b\rangle\rangle$, respectively. The lemmas then follow from his more general [Ahmad and Ohm 1995, Theorem 1.6].

Remark 3.12. One sees that Lemmas 3.8 and 3.9 hold for any strongly multiplicative form $\varphi$ as defined in [Lam 2005]. The work lies with anisotropic Pfister forms. The remaining strongly multiplicative forms are isotropic, hence their function fields are rational and both lemmas become trivial.

Proposition 3.10 enables us to compare the standard norm varieties for two equal symbols.

Corollary 3.13. The standard norm varieties $X\left(a_{1}, \ldots, a_{n}\right)$ and $X\left(b_{1}, \ldots, b_{n}\right)$ for $\left\{a_{1}, \ldots, a_{n}\right\}$ and $\left\{b_{1}, \ldots, b_{n}\right\}$ are birationally isomorphic if $\left\{a_{1}, \ldots, a_{n}\right\}=$ $\left\{b_{1}, \ldots, b_{n}\right\}$ in $K_{n}^{M}(k) / 2$.

Proof. By [Elman et al. 2008, Theorem 6.20], the two Pfister forms $\varphi=\left\langle\left\langle a_{1}, \ldots, a_{n}\right\rangle\right\rangle$ and $\varphi^{\prime}=\left\langle\left\langle b_{1}, \ldots, b_{n}\right\rangle\right\rangle$ are equivalent. Proposition 3.10 now implies their associated subforms $\psi$ and $\psi^{\prime}$ are birationally equivalent. By Theorem 3.7, $X\left(a_{1}, \ldots, a_{n}\right)$ and $X\left(b_{1}, \ldots, b_{n}\right)$ are birationally isomorphic.

Example 3.14. For any nonzero norm $N_{k\left(\sqrt{a_{i}}\right) / k}(u)$, we know

$$
\left\{a_{1}, \ldots, a_{i}, \ldots, a_{j}, \ldots, a_{n}\right\}=\left\{a_{1}, \ldots, a_{i}, \ldots, a_{j} N_{k\left(\sqrt{a_{i}}\right) / k}(u), \ldots, a_{n}\right\}
$$

in $K_{n}^{M}(k) / 2$. By Corollary 3.13, their standard norm varieties are birationally isomorphic. Or we can use Theorem 3.7 and Lemma 3.9, bypassing the chain P-equivalence theorem to see this as well.

\section{When $p>2$ and $n=2$}

When $p>2$ and $n=2$ we show that the standard norm varieties are birationally isomorphic to Severi-Brauer varieties.

Theorem 4.1. The standard norm variety $X(a, b)$ for $\{a, b\}$ is birationally isomorphic to the Severi-Brauer variety $\mathrm{SB}(A)$ associated to the cyclic algebra $A=$ $\left(a, b, \zeta_{p}\right)_{k}$.

Proof. Again, if we start the symmetric power construction with $X(a)=\operatorname{Spec}(L)$, where $L=k(\sqrt[p]{a})$, then $X(a, b)=Z\left(N_{L / k}-b\right)$ by Remark 2.5. We consider what happens in a split case, where $A_{L} \cong M_{p}(L)$ and $\operatorname{SB}\left(A_{L}\right) \cong \mathbb{P}_{L}^{p-1}$. Furthermore, 
if $G=\operatorname{Gal}(L / k)=\langle\sigma\rangle$ of order $p$ then over $L$, the norm $N_{L / k}(x)$ splits in to a product $\prod_{i=0}^{p-1} \sigma^{i}(x)$ for every $x \in L$. Define

$$
U_{L}=\left\{I \subset M_{p}(L)\right\},
$$

where

$$
I=\left\{\left(\begin{array}{cccc}
\alpha_{0} & 0 & \cdots & 0 \\
\vdots & \vdots & \ddots & \vdots \\
\alpha_{p-1} & 0 & \cdots & 0
\end{array}\right) M \mid \alpha_{i} \neq 0 \text { for all } i \text { and } M \in M_{p}(L)\right\} ;
$$

then $U_{L}$ is an open subset in $\mathrm{SB}\left(A_{L}\right)$ and we have a diagram

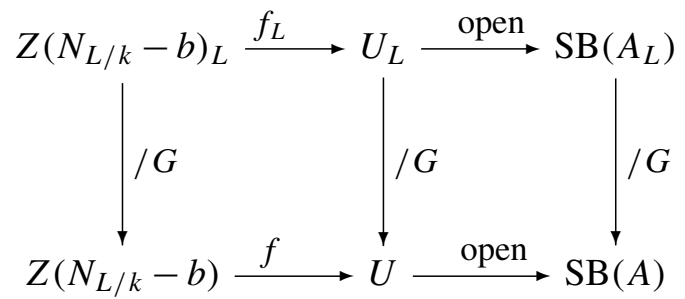

where $f_{L}$ can be described as

$$
\begin{aligned}
Z\left(N_{L / k}-b\right)_{L} & \stackrel{f_{L}}{\longrightarrow} U_{L}, \\
\left(x, \sigma(x), \ldots, \sigma^{p-1}(x)\right) & \longmapsto\left(x: x \sigma(x): \ldots: x \sigma(x) \cdots \sigma^{p-1}(x)\right),
\end{aligned}
$$

if we abuse notation and write points in $\mathrm{SB}\left(A_{L}\right)$ in projective coordinates. We verify that $f_{L}$ is $G$-equivariant:

$$
\begin{aligned}
f_{L}\left(\sigma \cdot\left(x, \sigma(x), \ldots, \sigma^{p-2}(x), \sigma^{p-1}(x)\right)\right) \\
\quad=f\left(\sigma(x), \sigma^{2}(x), \ldots, \sigma^{p-1}(x), \sigma^{p}(x)\right) \\
=\left(\sigma(x): \sigma(x) \sigma^{2}(x): \ldots: \sigma(x) \cdots \sigma^{p-1}(x): \sigma(x) \sigma^{2}(x) \cdots \sigma^{p}(x)\right) \\
\quad=\left(\sigma(x): \sigma(x) \sigma^{2}(x): \ldots: \sigma(x) \cdots \sigma^{p-1}(x): b\right),
\end{aligned}
$$

while

$$
\begin{aligned}
\sigma \cdot f_{L}\left(x, \sigma(x), \ldots, \sigma^{p-1}(x)\right) & \\
& =\left(\begin{array}{ccccc}
0 & 1 & 0 & \cdots & 0 \\
0 & 0 & 1 & \ddots & \vdots \\
\vdots & \vdots & \ddots & \ddots & 0 \\
0 & 0 & \cdots & 0 & 1 \\
b & 0 & \cdots & 0 & 0
\end{array}\right)\left(\begin{array}{c}
x \\
x \sigma(x) \\
\vdots \\
x \sigma(x) \cdots \sigma^{p-2}(x) \\
x \sigma(x) \cdots \sigma^{p-1}(x)
\end{array}\right) \\
& =\left(x \sigma(x): x \sigma(x) \sigma^{2}(x): \ldots: x \sigma(x) \cdots \sigma^{p-1}(x): b x\right) \\
& =\left(\sigma(x): \sigma(x) \sigma^{2}(x): \ldots: \sigma(x) \cdots \sigma^{p-1}(x): b\right) .
\end{aligned}
$$


In function fields, we have an isomorphism of the same name $f_{L}$ from $L\left(U_{L}\right)=$ $L\left(t_{1} / t_{0}, \ldots, t_{p} / t_{0}\right)$ to $L\left(Z\left(N_{L / k}-b\right)_{L}\right)=L\left(x, \sigma(x), \ldots, \sigma^{p-1}(x)\right)$,

$$
L\left(\frac{t_{1}}{t_{0}}, \ldots, \frac{t_{p}}{t_{0}}\right) \stackrel{f_{L}}{\longrightarrow} L\left(x, \sigma(x), \ldots, \sigma^{p-1}(x)\right), \quad \frac{t_{i}}{t_{0}} \mapsto x \sigma(x) \cdots \sigma^{i-1}(x),
$$

where $i=1, \ldots, p$ and $t_{p} / t_{0}=b$ with inverse

$$
L\left(x, \sigma(x), \ldots, \sigma^{p-1}(x)\right) \stackrel{f_{L}^{-1}}{\longrightarrow} L\left(\frac{t_{1}}{t_{0}}, \ldots, \frac{t_{p}}{t_{0}}\right), \quad \sigma^{i-1}(x) \mapsto \frac{t_{i}}{t_{i-1}} .
$$

We verify that $f_{L}$ respects the $G$-action:

$$
\begin{aligned}
f_{L}\left(\sigma \cdot \frac{t_{i}}{t_{0}}\right) & =f_{L}\left(\frac{t_{i+1}}{t_{1}}\right) \\
& =f_{L}\left(\left(\frac{t_{i+1}}{t_{0}}\right)\left(\frac{t_{1}}{t_{0}}\right)^{-1}\right) \\
& =x \sigma(x) \cdots \sigma^{i}(x) x^{-1} \\
& =\sigma(x) \cdots \sigma^{i}(x),
\end{aligned}
$$

while

$$
\sigma \cdot f_{L}\left(\frac{t_{i}}{t_{0}}\right)=\sigma \cdot\left(x \sigma(x) \cdots \sigma^{i-1}(x)\right)=\sigma(x) \cdots \sigma^{i}(x) .
$$

Therefore $Z\left(N_{L / k}-b\right)_{L}$ is birationally isomorphic to $U_{L}$. So $Z\left(N_{L / k}-b\right)$ is birationally isomorphic to $U$, hence to $\mathrm{SB}(A)$.

This theorem enables us to compare the standard norm varieties for two equal symbols.

Corollary 4.2. The standard norm varieties $X\left(a_{1}, a_{2}\right)$ and $X\left(b_{1}, b_{2}\right)$ for $\left\{a_{1}, a_{2}\right\}$ and $\left\{b_{1}, b_{2}\right\}$ are birationally isomorphic if $\left\{a_{1}, a_{2}\right\}=\left\{b_{1}, b_{2}\right\}$ in $K_{2}^{M}(k) / p$.

Proof. By the norm residue homomorphism $K_{2}^{M}(k) / p \rightarrow \operatorname{Br}_{p}(k)$, the classes of $\left(a_{1}, a_{2}, \zeta_{p}\right)_{k}$ and $\left(b_{1}, b_{2}, \zeta_{p}\right)_{k}$ are equal in the subgroup $\operatorname{Br}_{p}(k)$ of elements of exponent $p$ in the Brauer group $\operatorname{Br}(k)$. Since they have the same dimension, $\left(a_{1}, a_{2}, \zeta_{p}\right)_{k}$ and $\left(b_{1}, b_{2}, \zeta_{p}\right)_{k}$ are isomorphic as algebras. Hence

$$
\mathrm{SB}\left(\left(a_{1}, a_{2}, \zeta_{p}\right)_{k}\right) \cong \mathrm{SB}\left(\left(a_{1}, a_{2}, \zeta_{p}\right)_{k}\right) .
$$

It follows from the theorem that $X\left(a_{1}, a_{2}\right) \approx X\left(b_{1}, b_{2}\right)$.

\section{When $p>2$ and $n=3$}

When $p>2$ and $n=3$, we show that the standard norm varieties are birationally isomorphic to varieties defined by reduced norms of cyclic algebras. 
Theorem 5.1. The standard norm variety $X(a, b, c)$ for $\{a, b, c\}$ is birationally isomorphic to $\mathrm{Z}\left(\mathrm{Nrd}_{A / k}-c\right)$, where $A=\left(a, b, \zeta_{p}\right)_{k}$.

Proof. We consider what happens in a split case. Let $L=k(\sqrt[p]{a})$ and use $\mathrm{SB}(A)$ as the standard norm variety $X(a, b)$ for $\{a, b\}$. Once again, $A_{L} \cong M_{p}(L)$ and $\mathrm{SB}\left(A_{L}\right) \cong \mathbb{P}_{L}^{p-1}$. Our symmetric power construction looks like the front square over $k$ and the back square over $L$ :

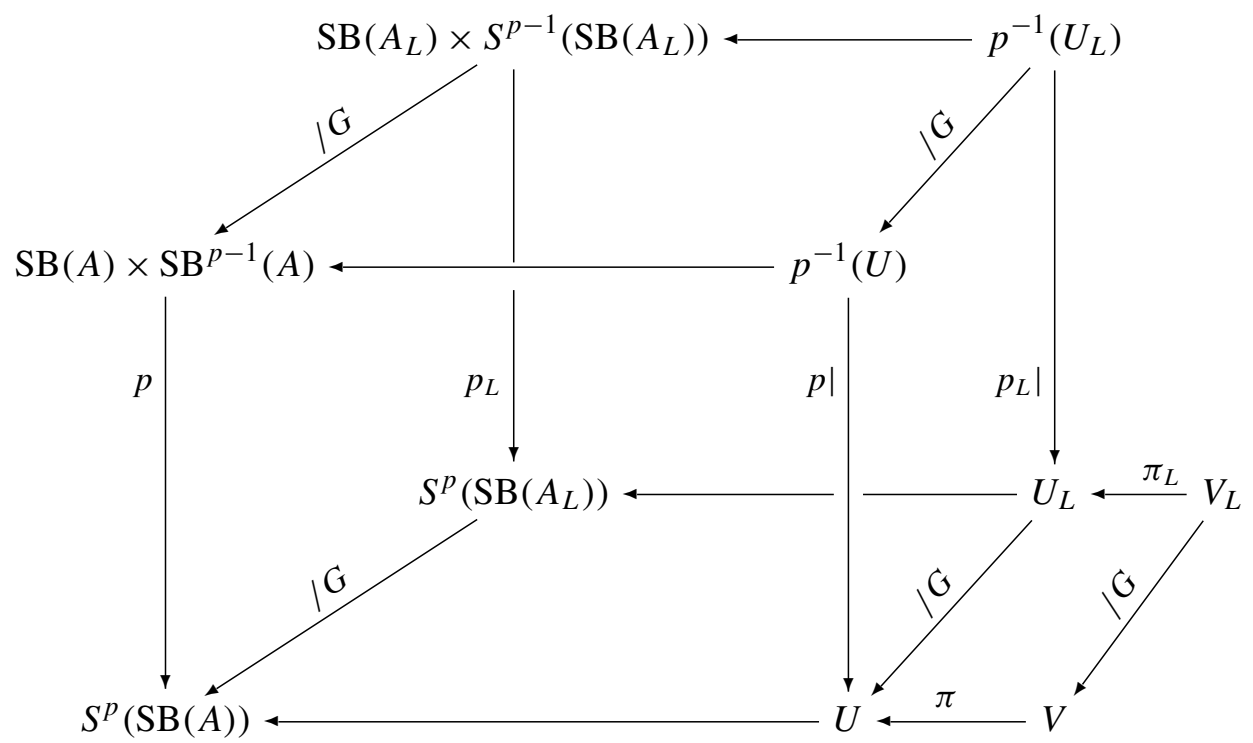

Now let $X_{L}$ denote the variety of all étale subalgebras of degree $p$ in $\operatorname{End}_{L}\left(L^{p}\right)$. If each subalgebra $D \in X_{L}$ is generated by a matrix $\lambda$, where $\lambda=\left(\lambda_{1}, \ldots, \lambda_{p}\right)$ is its diagonal form, then $S_{p}$ acts trivially on $X_{L}$ by permuting the diagonal entries. So we have an $S_{p}$-equivariant map

$$
U_{L} \stackrel{f_{L}}{\longrightarrow} X_{L}, \quad\left(u_{1}, \ldots, u_{p}\right) \mapsto D,
$$

where $D$ is the étale subalgebra whose eigenspaces are the lines $u_{1}, \ldots, u_{p}$, with inverse $f_{L}^{-1}: D \mapsto\left(u_{1}, \ldots, u_{p}\right)$. This map fits into the following commutative diagram:

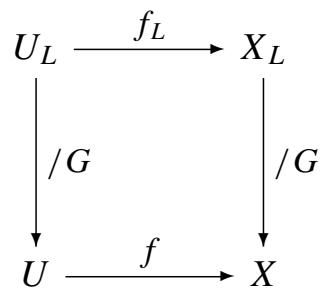


and we get vector bundles over the last diagram,

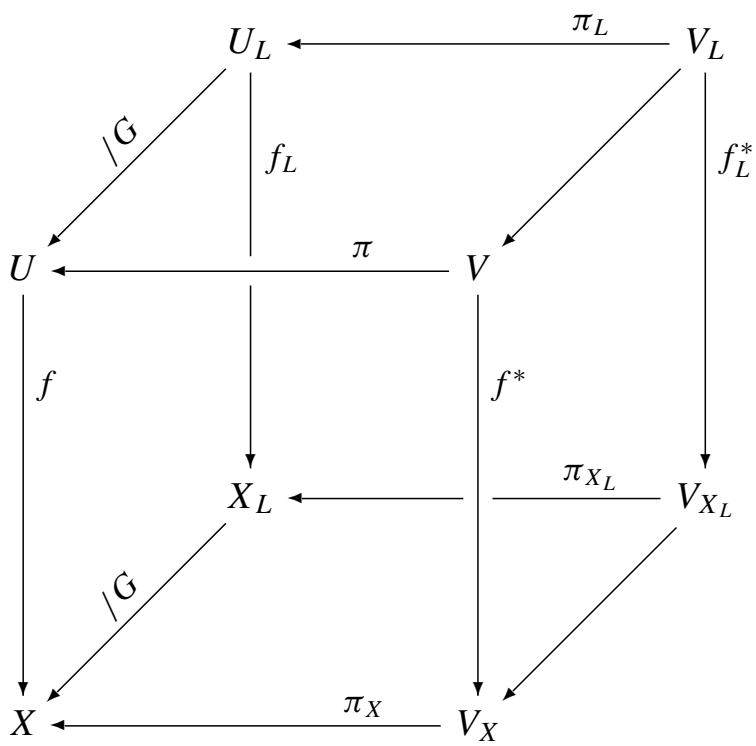

For each $\left(u_{1}, \ldots, u_{p}\right) \in U_{L}$, the preimage $p_{L}^{-1}\left(\left(u_{1}, \ldots, u_{p}\right)\right)$ consists of $p$ points $y_{1}, \ldots, y_{p}$, where each $y_{i}$ is of the form $\left(u_{i},\left(u_{1}, \ldots, \check{u}_{i}, \ldots, u_{p}\right)\right)$. So $\pi_{L}^{-1}\left(\left(u_{1}, \ldots, u_{p}\right)\right)=\left\{\left(\left(u_{1}, \ldots, u_{p}\right), x_{1}, \ldots, x_{p}\right) \mid x_{i} \in L\left(y_{i}\right)\right\}$. Correspondingly, $\pi_{X_{L}}^{-1}(D)=\{(D, d) \mid d \in D\}$. Both are algebras of rank $p$ over $L$. We can describe the back face of the cube pointwise:

$$
\begin{gathered}
\left(\left(u_{1}, \ldots, u_{p}\right), x_{1}, \ldots, x_{p}\right) \stackrel{f_{L}^{*}}{\longrightarrow} \\
\pi_{L} \downarrow
\end{gathered}\left(D,\left(\begin{array}{cccc}
x_{1} & 0 & \cdots & 0 \\
0 & \ddots & \ddots & \vdots \\
\vdots & \ddots & \ddots & 0 \\
0 & \cdots & 0 & x_{p}
\end{array}\right)\right)
$$

Note that if $q(t)=a_{1} t+\cdots+a_{p} t^{p}$ and $d=q(\lambda) \in D$ with eigenvalues $q\left(\lambda_{i}\right)$ then $f_{L}^{*^{-1}}(D, d)=\left(\left(u_{1}, \ldots, u_{p}\right), q\left(\lambda_{1}\right), \ldots, q\left(\lambda_{p}\right)\right)$.

Therefore, in $V_{L}$ and $V_{X_{L}}$ we have two birationally isomorphic subvarieties $Z(N-c)_{L}$ and $Z\left(\operatorname{Nrd}_{A_{L} / L}-c\right)$, since

$$
\begin{aligned}
Z(N-c)_{L} & =\left\{\left(\left(u_{1}, \ldots, u_{p}\right), x_{1}, \ldots, x_{p}\right) \mid x_{1} \cdots x_{p}=c\right\} \\
& \cong\left\{(D, d) \mid D \subset A_{L} \text { étale of rank } p \text { and } d \in D \text { with } N_{D / L}(d)=c\right\} \\
& =\left\{(D, d) \mid D \subset A_{L} \text { étale of rank } p \text { and } d \in D \text { with } \operatorname{Nrd}_{A_{L} / L}(d)=c\right\} \\
& \cong\left\{d \in A_{L} \mid\langle d\rangle \subset A_{L} \text { étale of rank } p \text { and } \operatorname{Nrd}_{A_{L} / L}(d)=c\right\} \\
& (\operatorname{via}(D, d) \mapsto d)
\end{aligned}
$$




$$
\begin{aligned}
& =\left\{d \in A_{L} \mid \operatorname{Nrd}_{A_{L} / L}(d)=c\right\} \\
& \qquad\left\{d \in A_{L} \mid \text { its minimal polynomial } m_{d}(t) \text { is of degree } p\right\} \\
& =\left\{d \in A_{L} \mid \operatorname{Nrd}_{A_{L} / L}(d)=c\right\} \\
& \qquad\left\{\left\{d \in A_{L} \mid x_{i} \neq x_{j} \text { for all of its eigenvalues } x_{i}, x_{j}\right\}\right. \\
& \approx\left\{d \in A_{L} \mid \operatorname{Nrd}_{A_{L} / L}(d)=c\right\} \\
& =Z\left(\operatorname{Nrd}_{A_{L} / L}-c\right) .
\end{aligned}
$$

Note that the intersection above is nonempty - it contains, for example, the diagonal matrix $\left(c / \zeta_{p}^{(p-1) / 2}, \zeta_{p}, \ldots, \zeta_{p}^{p-1}\right)$ - and the second set is open. Hence our standard norm variety $X(a, b, c)=Z(N-c)$ is birationally isomorphic to $Z\left(\operatorname{Nrd}_{A / k}-c\right)$ over $k$.

Knowing that $X(a, b, c)$ is birationally isomorphic to $Z\left(\operatorname{Nrd}_{A / k}-c\right)$, where $A=$ $\left(a, b, \zeta_{p}\right)_{k}$, may allow us to compare $X(a, b, c)$ and $X\left(a^{\prime}, b^{\prime}, c^{\prime}\right)$ when $\{a, b, c\}=$ $\left\{a^{\prime}, b^{\prime}, c^{\prime}\right\}$ in $K_{3}^{M}(k) / p$. If we know $Z\left(\operatorname{Nrd}_{A / k}-c\right) \approx Z\left(\operatorname{Nrd}_{A^{\prime} / k}-c^{\prime}\right)$, where $A^{\prime}=$ $\left(a^{\prime}, b^{\prime}, \zeta_{p}\right)_{k}$, then we can draw the same corollary for $p>2$ and $n=3$ as we did for $p=2$ in Corollary 3.13 and for $p>2$ and $n=2$ in Corollary 4.2.

\section{Acknowledgements}

This paper was my thesis. I thank my advisor Alexander Merkurjev for his guidance and my colleague Aaron Silberstein for his influence on submitting it for publication.

\section{References}

[Ahmad and Ohm 1995] H. Ahmad and J. Ohm, "Function fields of Pfister neighbors", J. Algebra 178:2 (1995), 653-664. MR 96k:11040 Zbl 0841.11015

[Elman and Lam 1972] R. Elman and T. Y. Lam, "Pfister forms and $K$-theory of fields", J. Algebra 23 (1972), 181-213. MR 46 \#1882 Zbl 0246.15029

[Elman et al. 2008] R. Elman, N. Karpenko, and A. S. Merkurjev, The algebraic and geometric theory of quadratic forms, Colloquium Publications 56, American Mathematical Society, Providence, RI, 2008. MR 2009d:11062 Zbl 1165.11042

[Haesemeyer and Weibel 2009] C. Haesemeyer and C. Weibel, "Norm varieties and the chain lemma (after Markus Rost)", pp. 95-130 in Algebraic topology (Oslo, 2007), edited by N. Baas et al., Abel Symposia 4, Springer, Berlin, 2009. MR 2011f:19002 Zbl 1244.19003

[Haesemeyer and Weibel 2016] C. Haesemeyer and C. Weibel, "The norm residue theorem in motivic cohomology", preprint, 2016, available at http://www.math.rutgers.edu/ weibel/BK.pdf.

[Karpenko and Merkurjev 2013] N. A. Karpenko and A. S. Merkurjev, "On standard norm varieties", Ann. Sci. Éc. Norm. Supér. (4) 46:1 (2013), 175-214. MR 3087392 Zbl 1275.14006

[Lam 2005] T. Y. Lam, Introduction to quadratic forms over fields, Graduate Studies in Mathematics 67, American Mathematical Society, Providence, RI, 2005. MR 2005h:11075 Zbl 1068.11023

[Milnor 1970] J. Milnor, "Algebraic K-theory and quadratic forms", Invent. Math. 9 (1970), 318344. MR 41 \#5465 Zbl 0199.55501 
[Suslin and Joukhovitski 2006] A. Suslin and S. Joukhovitski, "Norm varieties", J. Pure Appl. Algebra 206:1-2 (2006), 245-276. MR 2008a:14015 Zbl 1091.19002

[Voevodsky 2003] V. Voevodsky, "Motivic cohomology with $\mathbb{Z} / 2$-coefficients", Publ. Math. Inst. Hautes Études Sci. 98 (2003), 59-104. MR 2005b:14038b Zbl 1057.14028

[Voevodsky 2011] V. Voevodsky, "On motivic cohomology with $\mathbb{Z} /$ l-coefficients", Ann. of Math.

(2) 174:1 (2011), 401-438. MR 2012j:14030 Zbl 1236.14026

Received 26 Aug 2015. Revised 19 Oct 2015. Accepted 3 Nov 2015.

DinH HuU NGUYEN: dinhuun@gmail.com

Department of Mathematics, UCLA, Los Angeles, CA 90095, United States 


\title{
ANNALS OF K-THEORY
}

\author{
msp.org/akt
}

EDITORIAL BOARD

\author{
Paul Balmer University of California, Los Angeles, USA \\ balmer@math.ucla.edu \\ Spencer Bloch University of Chicago, USA \\ bloch@math.uchicago.edu \\ Alain Connes Collège de France; Institut des Hautes Études Scientifiques; Ohio State University \\ alain@connes.org \\ Guillermo Cortiñas Universidad de Buenos Aires and CONICET, Argentina \\ gcorti@dm.uba.ar \\ Eric Friedlander University of Southern California, USA \\ ericmf@usc.edu \\ Max Karoubi Institut de Mathématiques de Jussieu - Paris Rive Gauche, France \\ max.karoubi@imj-prg.fr \\ Gennadi Kasparov Vanderbilt University, USA \\ gennadi.kasparov@vanderbilt.edu \\ Alexander Merkurjev \\ University of California, Los Angeles, USA \\ merkurev@math.ucla.edu \\ Amnon Neeman amnon.Australian National University \\ neeman@anu.edu.au \\ Jonathan Rosenberg (Managing Editor) \\ University of Maryland, USA \\ jmr@math.umd.edu \\ Marco Schlichting University of Warwick, UK \\ schlichting@warwick.ac.uk \\ Andrei Suslin Northwestern University, USA \\ suslin@math.northwestern.edu \\ Vladimir Voevodsky Institute for Advanced Studies, USA \\ vladimir@math.ias.edu \\ Charles Weibel (Managing Editor) \\ Rutgers University, USA \\ weibel@math.rutgers.edu \\ Guoliang Yu Texas A\&M University, USA \\ guoliangyu@math.tamu.edu \\ PRODUCTION \\ $\begin{array}{ll}\text { Silvio Levy } & \text { (Scientific Editor) } \\ \text { production@msp.org }\end{array}$
}

Annals of K-Theory is a journal of the K-Theory Foundation (ktheoryfoundation.org). The K-Theory Foundation acknowledges the precious support of Foundation Compositio Mathematica, whose help has been instrumental in the launch of the Annals of K-Theory.

See inside back cover or msp.org/ant for submission instructions.

The subscription price for 2016 is US $\$ 400 /$ year for the electronic version, and $\$ 450 /$ year (+\$25, if shipping outside the US) for print and electronic. Subscriptions, requests for back issues and changes of subscribers address should be sent to MSP.

Annals of K-Theory (ISSN 2379-1681 electronic, 2379-1683 printed) at Mathematical Sciences Publishers, 798 Evans Hall \#3840, c/o University of California, Berkeley, CA 94720-3840 is published continuously online. Periodical rate postage paid at Berkeley, CA 94704, and additional mailing offices.

AKT peer review and production are managed by EditFlow ${ }^{\circledR}$ from MSP.

PUBLISHED BY

mathematical sciences publishers nonprofit scientific publishing

http://msp.org/

(C) 2016 Mathematical Sciences Publishers 


\section{ANNALS \\ OF \\ K-THEORY}

2016

no. 4

Splitting the relative assembly map, Nil-terms and involutions

Wolfgang Lück and Wolfgang Steimle

Birational motives, I: pure birational motives

Bruno Kahn and Ramdorai Sujatha

On the $K$-theory of linear groups

Daniel Kasprowski

Standard norm varieties for Milnor symbols $\bmod p$

Dinh Huu Nguyen 